A proposito di immagini patognomoniche:

un caso infrequente di encefalopatia acuta

\title{
About pathognomonic images: an infrequent case of acute encephalopathy
}

\author{
Alessandro Grasso ${ }^{*}$, Pietro Nurra ${ }^{1}$, Luigia Cavallo², Giorgio Menardo \\ 1 U O Medicina Interna e Gastroenterologia, Ospedale "San Paolo", Savona \\ 2 U O Radiologia, Ospedale "San Paolo", Savona
}

\section{KEY WORDS}

Acute encephalopathy Thiamine deficiency

MRI

\begin{abstract}
Summary BACKGROUND The occurrence of acute encephalopathy is a dramatic clinical dilemma when usual diagnostic techniques (blood tests, cerebral CT and cerebrospinal fluid analysis) show no abnormalities. CLINICAL CASE We describe a case of a 73 years old man admitted in our Internal Medicine Unit for acute diarrhoea with vomiting and fever who developed a prolonged gastrointestinal dysmotility syndrome with poor nutritional intake. Although a parenteral support was provided, he developed acute encephalopathy followed by hypotension and lactic acidosis without evidence of renal and hepatic disease or glycemic alterations. Likewise, no cerebral CT and cerebrospinal fluid alterations were found. Conversely, cerebral MRI showed marked and diffuse DP-2 and FLAIR hyperintensity of the mesencephalic tectal plate, of the periaqueductal area, and of the periventricular region of the third ventricle including the median thalamic area. These MRI descriptions were considered pathognomonic of Wernicke encephalopathy. Thus, the immediate use of ev thiamine was followed by a prompt and complete recovery of neurological, hemodinamic and metabolic conditions. Conclusıons Non-alcoholic Wernicke encephalopathy is a rare and dramatic clinical event with high mortality. In this context, brain MRI is the best diagnostic tool providing a typical picture.
\end{abstract}

\section{Introduzione}

L'insorgenza di encefalopatia acuta costituisce un'emergenza medica che spesso sottende condizioni cliniche severe e complesse. La diagnostica tradizionale in urgenza (laboratorio, neuroimaging, rachicentesi) fornisce quasi sempre un aiuto diagnostico decisivo. Tuttavia, nelle rare circostanze in cui non si è in presenza di un danno cerebrale ischemico, emorragico, infettivo, né di un'encefalopatia metabolica, la soluzione sembra ardua. Nel caso che descriviamo, la specificità di alcuni segni radiologici ha permesso di risolvere in modo decisivo e inaspettatamente semplice una situazione diventata drammatica.

\footnotetext{
* Corrispondenza:

Alessandro Grasso, UO Medicina Interna e Gastroenterologia, Ospedale San Paolo, via Genova 38, 17100 Savona,

e-mail: a.grasso@asl2.liguria.it
}

\section{Caso clinico}

F.V, maschio, di 73 anni, senza precedenti clinici significativi in anamnesi, giunse alla nostra osservazione per la persistenza da circa una settimana di febbre intermittente con nausea, vomito e diarrea.

Pur in assenza di una diagnosi precisa (e avendo comunque escluso le cause più comuni di tossinfezione alimentare, colite infettiva o infiammatoria e la celiachia) e a causa del peggioramento dei sintomi gastroenterici e dello stato nutrizionale, venne inserito un supporto nutrizionale parenterale periferico proseguendo la terapia sintomatica. Sebbene le condizioni generali fossero rimaste a lungo scadenti, in ventesima giornata si assistette a un lentissimo miglioramento clinico con ripresa, seppur parziale, dell'alimentazione per via naturale.

Quando il paziente appariva ormai quasi completamente ristabilito, in trentesima giornata si manifestò un calo del visus bilaterale seguito, il giorno successivo, da rallentamento ideomotorio. Una tomografia computerizzata (TC) 
cerebrale risultava nella norma, a esclusione di un lieve ampliamento degli spazi subaracnoidei. Nella norma ammoniemia, glicemia, creatininemia, azotemia, sodiemia, potassiemia, calcemia, proteine totali, pH ematico e saturazione dell' $\mathrm{O}_{2}$. Nelle successive 24 ore lo stato di coscienza peggiorò, con comparsa di confusione, fino a sfociare in uno stato di coma conclamato associato a ipotensione e acidosi lattica. Anche la rachicentesi risultava priva di elementi patologici.

Si decise, infine, di effettuare una risonanza magnetica nucleare (RMN) cerebrale, che evidenziò una marcata e diffusa iperintensità di segnale in DP-T2 e FLAIR (Fluid-Attenuated Inversion Recovery) a livello periacqueduttale, tutt'attorno alle pareti del terzo ventricolo, in corrispondenza dei settori mediali del talamo e a livello del tetto mesencefalico. Le alterazioni descritte erano bilaterali e simmetriche e non si associavano a segni di effetto massa (Fig. 1). II reperto RMN fu giudicato dal radiologo tipico di encefalopatia di Wernicke. La somministrazione immediata di tiamina per via endovenosa (100 mg/die) indusse, in meno di 48 ore, un completo recupero dello stato di coscienza e in una settimana il paziente ritornò in uno stato di quasi completa autosufficienza.

\section{Discussione}

Sebbene l'encefalopatia di Wernicke (EW), una forma di encefalopatia acuta secondaria a deficit di tiamina, sia una manifestazione neurologica tipica del paziente alcolista, è stata descritta anche in non alcolisti. Secondo una recente revisione sistematica [1], la prevalenza in soggetti non alcolisti dell'EW e della sua variante sistemica caratterizzata da malattia cardiovascolare e metabolica, il beri beri (BB), sarebbe largamente sottostimata.

EW e BB sono state descritte principalmente come casi aneddotici dopo ingestione di pesce crudo contaminato con tiaminasi microbiche, in pazienti con malattie gastroenteriche associate a malassorbimento (morbo di Crohn, colite ulcerosa, intestino corto), pancreatite acuta, dopo chirurgia per l'obesità (chirurgia bariatrica), in corso di iperemesi gravidica. Inoltre sono state descritte in soggetti con neoplasia in fase avanzata, in pazienti emodializzati e durante nutrizione parenterale totale (NPT) senza supporto vitaminico. Questi ultimi casi rappresentano la serie più numerosa e, in effetti, anche in tutte le patologie suddette in cui è stata descritta EW-BB (forse con la sola eccezione dell'emodialisi, in cui è ipotizzato anche un meccanismo di deplezione della tiamina, vitamina idrosolubile), vi è quasi sempre la mancata supplementazione con tiamina delle sacche nutrizionali, con un tempo medio di comparsa dell'encefalopatia acuta che varia da 4 a 40 giorni dopo I'inizio della NPT [2]. In tali casi sono spesso riportate, oltre all'encefalopatia acuta, sia ipotensione arteriosa non responsiva alle misure antishock, sia acidosi lattica non responsiva alla somministrazione di bicarbonati [3]. Oltre alI'EW-BB classici, un deficit di tiamina è stato altresì documentato nel $40 \%$ degli anziani istituzionalizzati ospedaliz-
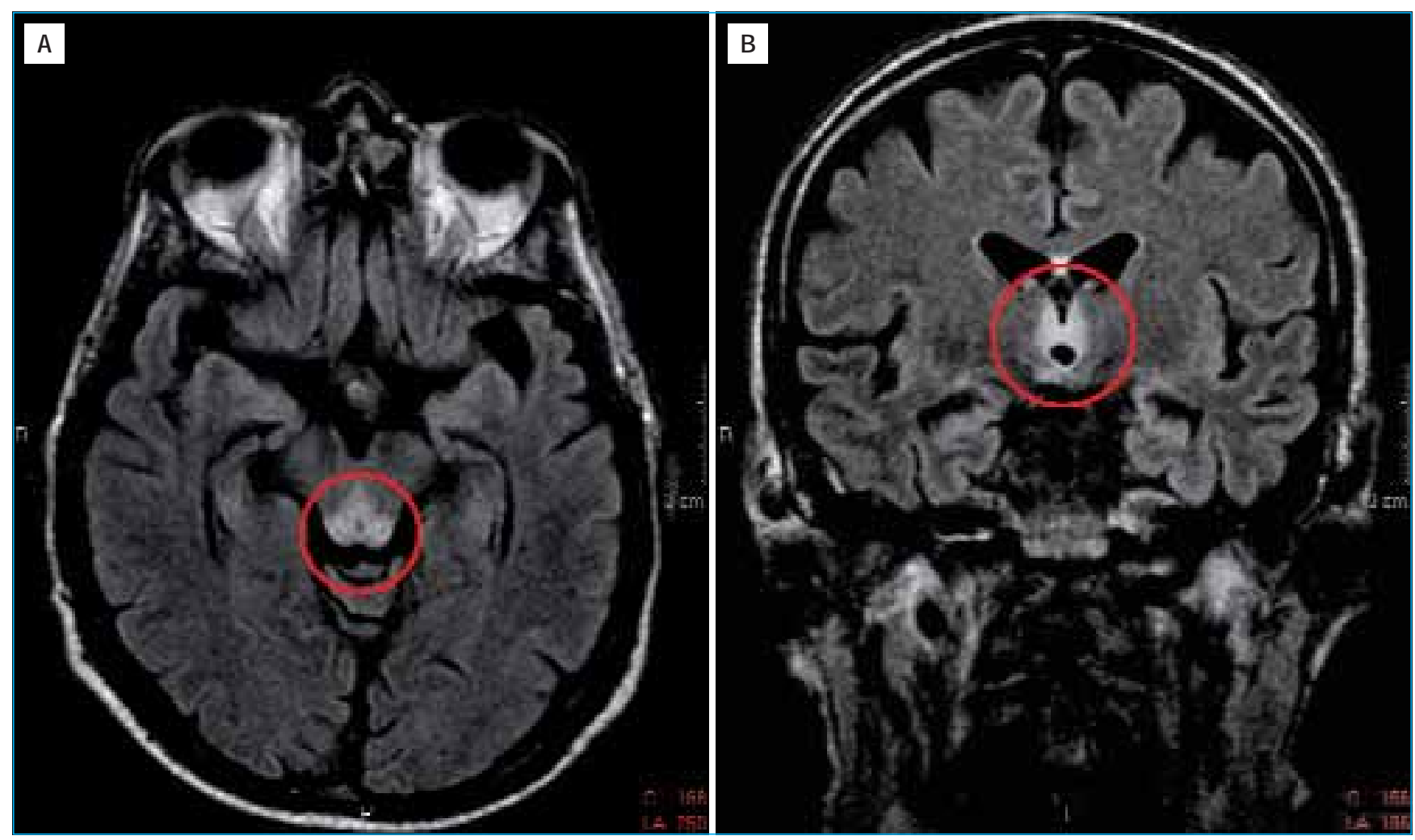

Figura 1 Sequenza coronale (A) e assiale FLAIR (B) con evidenza di alterazione di segnale a carico della sostanza periacqueduttale e periventricolare (cerchio rosso) 
zati [4] e nel 30\% circa dei pazienti con scompenso cardiaco ospedalizzati (a causa delle perdite urinarie di tiamina indotte da diuretico), nei quali può causare un peggioramento dei sintomi dello scompenso cardiaco [5].

Nel nostro paziente, la mancata supplementazione con tiamina della sacca nutrizionale è stata certamente cruciale nell'indurre encefalopatia acuta, benché non si possa escludere che una patologia flogistica o linfoproliferativa del tenue (unico tratto del tubo digerente che non è stato adeguatamente studiato), oltre a spiegare almeno in parte il quadro clinico d'esordio, possa aver partecipato al deficit vitaminico stesso.

Nell'EW, come riportato in letteratura, la TC non ha potere diagnostico, al contrario della RMN cerebrale. Quest'ultima presenta elementi che sono giudicati patognomonici, quali le aree di incrementato segnale nelle sequenze T2 pesate e FLAIR che circondano simmetricamente I'acquedotto, il terzo ventricolo, il pavimento del quarto ventricolo, i corpi mammillari, la parte mediale dei talami e la porzione superiore del nucleo caudato $[6,7]$. Tali alterazioni sono reversibili, come ha dimostrato un controllo RM N a 6 mesi (Fig. 2).

\section{Conclusioni}

L'encefalopatia acuta di Wernicke non alcol-correlata rappresenta una diagnosi infrequente e difficile. La possibilità di riconoscere alcuni elementi neuroradiologici specifici permette di recuperare in maniera estremamente semplice situazioni cliniche talora apparentemente irrimediabili. Occorre ricordare, in proposito, come in questo contesto la TC possieda una scarsa resa diagnostica e, pertanto, nel sospetto clinico è necessaria l'esecuzione di una RM N. Infine, in pazienti sottoposti a NPT per periodi prolungati, va tenuta presente l'esigenza di supplementare i nutrienti usati per la copertura del fabbisogno calorico con oligoelementi e vitamine, non tralasciando, in tale contesto, la vitamina B1.

\section{Bibliografia}

[1] Sechi G, Serra A. Wernicke's encephalopathy: new clinical settings and recent advances in diagnosis and management. Lancet Neurol 2007;6(5):442-55.

[2] Kitamura K, Yamaguchi T, Tanaka H, Hashimoto S, Yang M,

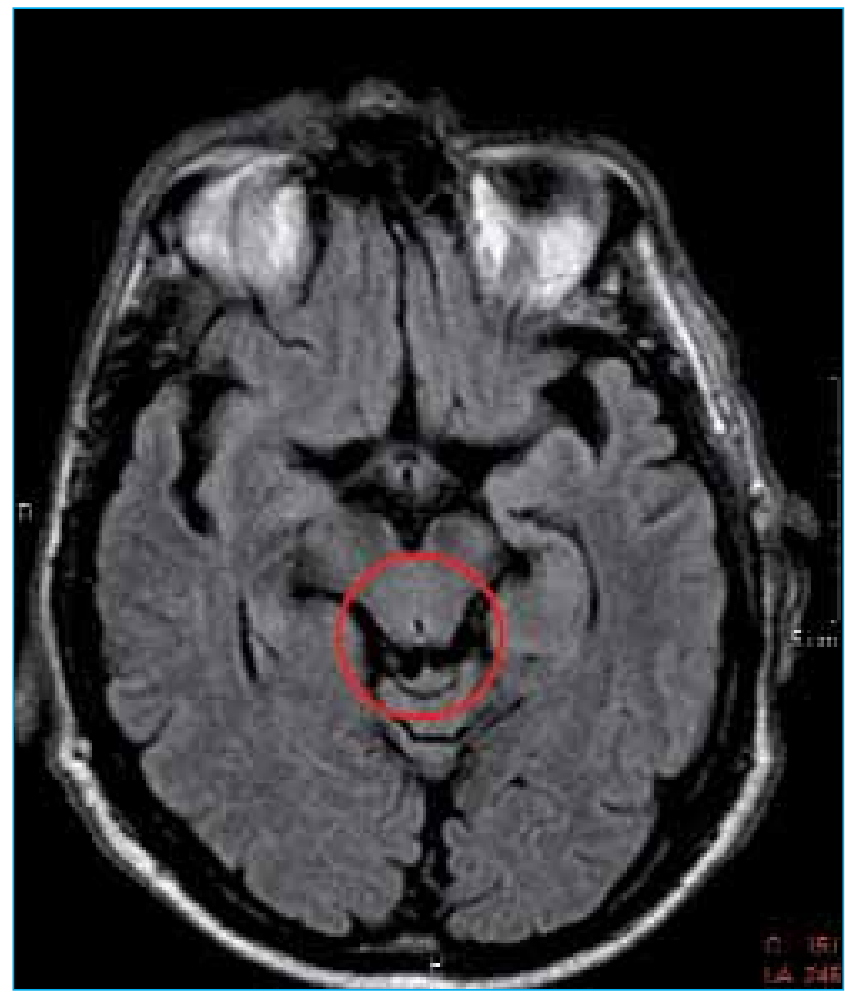

Figura 2 Sequenza assiale FLAIR. Controllo a 6 mesi con evidenza di completa scomparsa (cerchio rosso) dell'alterazione di segnale in sede periacqueduttale e periventricolare

Takahashi T. TPN-induced fulminant beriberi: a report on our experience and a review of the literature. Surg Today 1996; 26(10):769-76.

[3] Nakasaki H, Ohta M, Soeda J, et al. Clinical and biochemical aspects of thiamine treatment for metabolic acidosis during total parenteral nutrition. Nutrition 1997;13(2):110-7.

[4] Pepersack T, Garbusinski J , Robberecht J , Beyer I, Willems D, Fuss M. Clinical relevance of thiamine status amongst hospitalized elderly patients. Gerontology 1999;45(2):96-101.

[5] Hanninen SA, Darling PB, Sole MJ, Barr A, Keith ME. The prevalence of thiamine deficiency in hospitalized patients with congestive heart failure. J Am Coll Cardiol 2006;47(2): 354-61.

[6] Weidauer S, Nichtweiss M, Lanfermann H, Zanella FE. Wernicke encephalopathy: MR findings and clinical presentation. Eur Radiol 2003;13(5):1001-9.

[7] Zhong C, J in L, Fei G. MR imaging of nonalcoholic Wernicke encephalopathy: a follow-up study. AJ NR Am J Neuroradiol 2005;26(9):2301-5. 